Check for updates

Cite this: Phys. Chem. Chem. Phys., 2019, 21, 760

Received 6th June 2018,

Accepted 8th December 2018

DOI: $10.1039 / c 8 c p 03581 d$

rsc.li/pccp

\section{Magnetic structure of $\mathrm{UO}_{2}$ and $\mathrm{NpO}_{2}$ by first-principle methods $\$$.}

\author{
James T. Pegg, (D)*ab Ashley E. Shields, (D) ${ }^{\mathrm{c}}$ Mark T. Storr, ${ }^{\mathrm{b}}$ Andrew S. Wills, ${ }^{\mathrm{a}}$ \\ David O. Scanlon (D) ade and Nora H. de Leeuw (D) af
}

\begin{abstract}
The magnetic structure of the actinide dioxides $\left(\mathrm{AnO}_{2}\right)$ remains a field of intense research. A low-temperature experimental investigation of the magnetic ground-state is complicated by thermal energy released from the radioactive decay of the actinide nuclei. To establish the magnetic ground-state, we have employed high-accuracy computational methods to systematically probe different magnetic structures. A transverse $1 \mathrm{k}$ antiferromagnetic ground-state with $\mathrm{Fmmm}$ (No. 69) crystal symmetry has been established for $\mathrm{UO}_{2}$, whereas a ferromagnetic (111) ground-state with $R \overline{3} m$ (No. 166) has been established for $\mathrm{NpO}_{2}$. Band structure calculations have been performed to analyse these results.
\end{abstract}

\section{Introduction}

The magnetic ground-state of the actinide dioxides $\left(\mathrm{AnO}_{2}\right)$ is key to the design of reliable computational models. ${ }^{1}$ The major actinides $(\mathrm{An}=\mathrm{U}, \mathrm{Np}, \mathrm{Pu})$ are challenging systems to study. The low-temperature characterisation of the $\mathrm{AnO}_{2}$ magnetic groundstate is complicated by: the toxicity of the metals, ${ }^{2-4}$ nucleonic radioactive decay,,$^{2-4}$ and the inhomogeneity of samples. ${ }^{5-10}$ To compensate for known radiogenic and experimental issues, computational methods offer a complementary means of investigation.

A number of experimental studies on the $\mathrm{AnO}_{2}$ indicate $F m \overline{3} m$ (No. 225) cubic symmetry, ${ }^{11,12}$ where the $\mathrm{An}^{4+}$ cations occupy octahedral (4a) sites and the $\mathrm{O}^{2-}$ anions occupy tetrahedral (8c) sites. Low-temperature measurements of $\mathrm{UO}_{2}$ have indicated the $P a \overline{3}$ (No. 205) crystal symmetry, which involves an internal

\footnotetext{
${ }^{a}$ Department of Chemistry, University College London, 20 Gordon Street, London WC1H OAJ, UK. E-mail: uccajtp@ucl.ac.uk

${ }^{b}$ Atomic Weapons Establishment (AWE) Plc, Aldermaston, Reading, RG7 4PR, UK

${ }^{c}$ Oak Ridge National Laboratory, One Bethel Valley Road, Oak Ridge, Tennessee 37831, USA

${ }^{d}$ Diamond Light Source Ltd., Diamond House, Harwell Science and Innovation Campus, Didcot, Oxfordshire OX11 ODE, UK

${ }^{e}$ Thomas Young Centre, University College London, Gower Street, London WC1E 6BT, UK

${ }^{f}$ Cardiff University, School of Chemistry, Main Building, Park Place, Cardiff, CF1D 3AT, UK

$\dagger$ Electronic supplementary information (ESI) available. See DOI: 10.1039/c8cp03581d \# This manuscript has been co-authored by UT-Battelle, LLC, under contract DE-AC05-00OR22725 with the US Department of Energy (DOE). The US government retains and the publisher, by accepting the article for publication, acknowledges that the US government retains a nonexclusive, paid-up, irrevocable, worldwide license to publish or reproduce the published form of this manuscript, or allow others to do so, for US government purposes. DOE will provide public access to these results of federally sponsored research in accordance with the DOE Public Access Plan (http://energy.gov/downloads/doe-public-access-plan).
}

distortion of the $\mathrm{O}^{2-}$ ions within the cubic lattice. ${ }^{13}$ The magnetic structure of the $\mathrm{AnO}_{2}$ is often inferred by the one-electron crystal electric field (Fig. 1). In crystal-field (CF) theory, the $5 f$ electrons are highly localized due to the insulating nature of these materials. Thus, the orbitals do not hybridize and the crystal field is influenced by the electrostatic potential. Therefore low-spin coupling is initially considered, with the crystal field as a perturbation. The spin-orbit interaction (SOI) ${ }^{14}$ generates $j=7 / 2$ and $j=5 / 2$ electronic levels, whereby the degeneracy of the levels is further broken by the crystal field. The interpretation of the magnetic structure by CF theory is only valid for the one-electron case, ${ }^{15,16}$ whereas the magnetic structure of the $\mathrm{AnO}_{2}$ involves the complex interplay of spin-lattice, magneto-elastic, super-exchange, multipolar and cooperative JahnTeller interactions. ${ }^{17-21}$ The type of magnetism can be classified into paramagnetic (PM); diamagnetic (DM); ferromagnetic (FM); anti-ferromagnetic (AFM); and ferrimagnetic (FI) behaviour. ${ }^{1}$

In DM materials, as all electrons are paired, no magnetic moments are associated with the individual ions and as a result the net magnetic moment of the crystal is zero. The only magnetic response is a weak repulsion in an applied magnetic field. In ordered FM, AFM or FI materials, a magnetic moment is formed by unpaired electrons. These magnetic moments may couple resulting in a periodic arrangement. If the magnetic moments are aligned in one dimension, the material is FM and has a net crystal moment. The direction of the magnetic moment may vary, resulting in FM (111), (011) or (001) states in the $F m \overline{3} m$ crystal. If the magnetic moments are opposed, the material is AFM and no net magnetic moment exists. In the propagation vector formulism, contributions can be combined from a number of symmetry-related wavevectors. These are termed multi-k structures. ${ }^{1}$ In the following article, single $(1 \mathrm{k})$, double (2k), and triple (3k) structures have been considered.

If the opposing magnetic moments on the ions are unequal, the material is FI and the crystal has a net magnetic moment. 


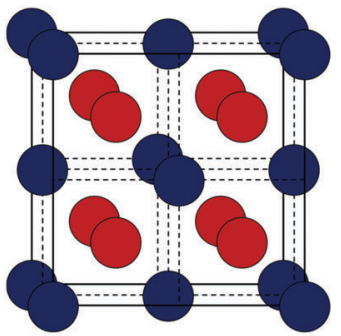

Diamagnetic

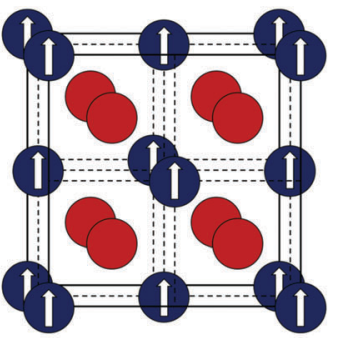

Ferromagnetic (001)

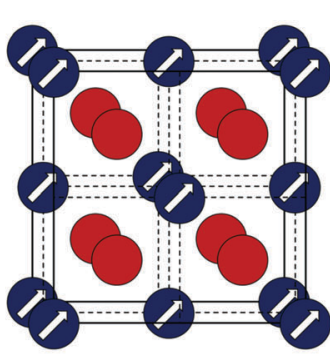

Ferromagnetic (011 or 111)

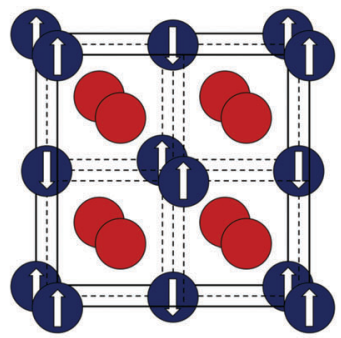

Transverse $1 \mathrm{k}$

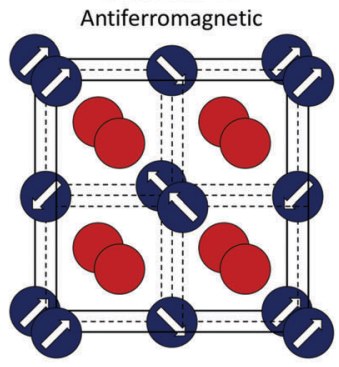

Longitudinal (2 or 3 )k Antiferromagnetic

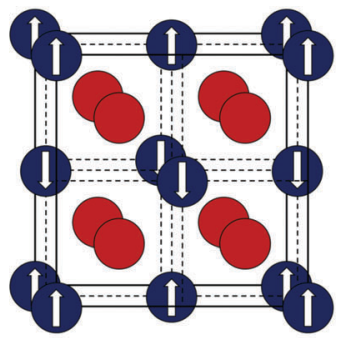

Longitudinal $1 \mathrm{k}$ Antiferromagnetic

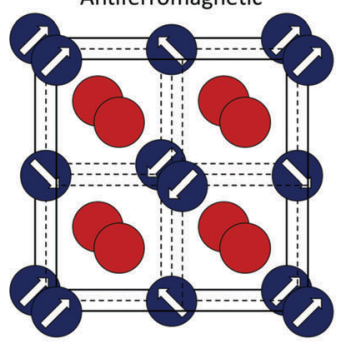

Transverse (2 or 3 )k Antiferromagnetic

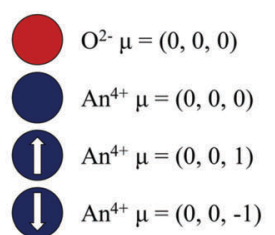

(7) $\mathrm{An}^{4+} \mu=(0,1,1)$

or $\mu=(1,1,1)$

$\mathrm{An}^{4+} \mu=(0,-1,1)$

or $\mu=(-1,-1,1)$

$\mathrm{An}^{4+} \mu=(0,1,-1)$

or $\mu=(-1,1,-1)$

$\mathrm{An}^{4+} \mu=(0,-1,-1)$

or $\mu=(1,-1,-1)$

Fig. 1 Illustration of magnetic configurations in the calcium fluorite $\left(\mathrm{CaF}_{2}\right)$ crystal structure.

If the magnetic moments are decoupled, the material is PM and there is no ordered distribution. The net magnetic moment of the crystal will average to zero. In addition, an isolated ion is said to be PM if it has a magnetic moment. ${ }^{22}$ As the magnetic moment of the $\mathrm{An}^{4+}$ ions (in stoichiometric $\mathrm{AnO}_{2}$ ) are equal (due to the occupation of chemically equivalent sites) the FI state cannot exist. In addition, significant exchange interactions are expected to be present and cause magnetic order. As a result, neither FI or PM is considered in this study. The manifestation of metastable states and the juxtaposition of energy levels makes the determination of the magnetic ground-state challenging. In this paper, the magnetic structures of the $\mathrm{AnO}_{2}$ are calculated for multiple configurations (Table 1).

The magnetic wavevectors are directed along the main axes of the crystal unit cell. The final magnetic moment of each ion is calculated using the Pythagorean theorem in eqn (1).

$$
\left\|\mu^{2}\right\|=\sum_{k=1}^{3}\left\|\mu_{k}^{2}\right\|
$$

The magnitude of the vector $(\mu)$ is calculated from its orthogonal $\left(\mu_{k}\right)$ components. To correctly access the noncollinear 2-3k AFM states, SOI must be considered.

The crystal structure is coupled by $\mathrm{SOI}^{14}$ to the magnetic state. In the high-temperature PM state, the $F m \overline{3} m$ (No. 255) crystal structure is stabilized by the intrinsic magnetic disorder (Fig. 2). At low-temperature $(T=30.8 \mathrm{~K})$, the ordered FM and AFM configurations can cause a crystallographic distortion due to an imbalance of magnetic forces. The extent of the disruption is dependent upon the magnitude of the magnetic forces within the crystal. Low-temperature $(T<30.8 \mathrm{~K})$ measurements of the $\mathrm{AnO}_{2}$ magnetic ground-state are extremely challenging, due to: thermal energy generated by the radioactive decay of the actinide nuclei, the inhomogeneity of samples and high chemical sensitivity to environmental conditions. ${ }^{23-30}$ In this paper, the low-temperature magnetic structure of $\mathrm{UO}_{2}$ and $\mathrm{NpO}_{2}$ is investigated using firstprinciple methods. In addition, a computationally tractable method for the inclusion of magnetic order in the $\mathrm{AnO}_{2}$ has been developed that can be applied to larger systems. ${ }^{132}$

A key difficulty in the computational investigation of the $\mathrm{AnO}_{2}$ is the treatment of: relativistic influences, electron-correlation, and complex magnetic structures. The correct electronic structure of the $\mathrm{AnO}_{2}$ cannot be calculated by conventional density functional theory (DFT) based methods, ${ }^{31,32}$ due to the high degree of electroncorrelation, ${ }^{33}$ which often manifests as an underestimation of the electronic band-gap. ${ }^{9,34}$ To compensate, numerous methods have been developed such as, the self-interaction correction (SIC) method, ${ }^{35}$ modified density functional theory $(\mathrm{DFT}+U),{ }^{31,32,36-38}$ dynamic mean field theory (DMFT), ${ }^{39}$ and hybrid density functionals. ${ }^{40-42}$ Of these methods, DFT $+U$ offers a computationally

Table 1 The wave vectors for the ordered magnetic states

\begin{tabular}{|c|c|c|c|c|c|c|c|c|c|}
\hline Ion & \multicolumn{3}{|c|}{ Ferromagnetic } & \multicolumn{3}{|c|}{ Antiferromagnetic (longitudinal domain) } & \multicolumn{3}{|c|}{ Antiferromagnetic (transverse domain) } \\
\hline$(0,0,0)$ & $(0,0,1)$ & $(0,1,1)$ & $(1,1,1)$ & $(0,0,1)$ & $(0,1,1)$ & $(1,1,1)$ & $(0,0,1)$ & $(0,1,1)$ & $(1,1,1)$ \\
\hline$\left(\frac{1}{2}, 0, \frac{1}{2}\right)$ & $(0,0,1)$ & $(0,1,1)$ & $(1,1,1)$ & $(0,0, \overline{1})$ & $(0,1, \overline{1})$ & $(\overline{1}, 1, \overline{1})$ & $(0,0,1)$ & $(0, \overline{1}, 1)$ & $(\overline{1}, \overline{1}, 1)$ \\
\hline$\left(0, \frac{1}{2}, \frac{1}{2}\right)$ & $(0,0,1)$ & $(0,1,1)$ & $(1,1,1)$ & $(0,0, \overline{1})$ & $(0, \overline{1}, \overline{1})$ & $(1, \overline{1}, \overline{1})$ & $(0,0, \overline{1})$ & $(0,1, \overline{1})$ & $(\overline{1}, 1, \overline{1})$ \\
\hline
\end{tabular}



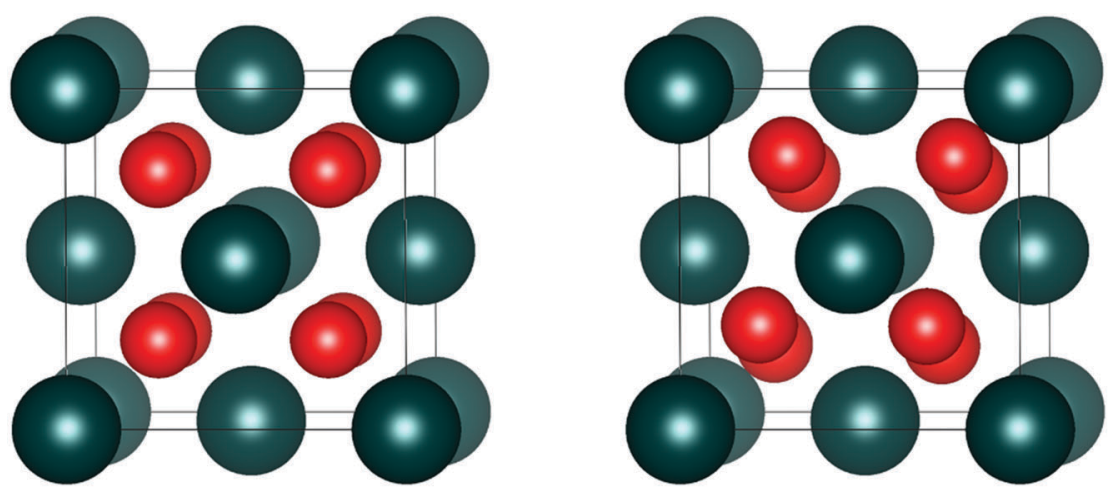

Fig. 2 Illustration of the actinide dioxide $\left(\mathrm{AnO}_{2}\right)$ crystal structure: (left) cubic $\mathrm{Fm} \overline{3} \mathrm{~m}$ symmetry, (right) cubic $\mathrm{Pa} \overline{\overline{3}}$ symmetry. The colours in the parentheses indicate the $\mathrm{An}^{4+}$ (blue) and $\mathrm{O}^{2-}$ (red) ions.

tractable means of investigation by treating the on-site Coulomb repulsion of the f-electrons with tuneable $U$ and $J$ modifiers, essentially adding an energy penalty to partial occupation of what should be localized f-electrons. The ground-state characteristics of f-electron compounds can be captured by $\mathrm{DFT}+U$ when SOI are included. ${ }^{43}$ By comparison, hybrid functionals offer one of the more accurate, but computationally highly expensive methods. The computationally intense nature of DMFT has been highlighted by a number of authors, but even this method has incorrectly calculated the early $\mathrm{AnO}_{2}$ as charge-transfer insulators. ${ }^{39,44-47}$ In addition to the highly-correlated and relativistic nature of actinide compounds, the identification and modelling of the magnetic ground-state is incredibly challenging. A limited number of publications have investigated noncollinear contributions, ${ }^{48-50}$ and collinear 1k AFM order is often used to model the electronic structure. ${ }^{9,34,42,49,51-63}$ The inclusion of spin-orbit interactions $(\mathrm{SOI})^{14}$ is often ignored, ${ }^{34,46,48,60-62,64}$ although a number of investigations have highlighted its importance. ${ }^{33,48}$ This is mainly due to the computational cost. ${ }^{63}$

The magnetic structure of $\mathrm{UO}_{2}$ displays interesting characteristics. In the Russell-Saunders (low-spin) coupling scheme, the ground-state of the $\mathrm{U}^{4+}$ ion is a ${ }^{3} \mathrm{H}_{4}\left(\Gamma_{5}\right.$ triplet $)$ multiplet. ${ }^{15,16,48}$ Low-temperature measurements of $\left(\mathrm{U}_{1-x} \mathrm{Th}_{x}\right) \mathrm{O}_{2}$ confirm a $\Gamma_{5}$ triplet magnetic ground-state. ${ }^{65}$ A discontinuous first-order phase transition (Néel temperature, $T_{\mathrm{N}}=30.8 \mathrm{~K}^{66}$ ) that is indicative of magnetic order has been confirmed by: heat capacity, ${ }^{67,68}$ magnetic susceptibility ${ }^{5}$ and neutron diffraction ${ }^{69-71}$ measurements. The nature of the magnetic ground-state has been investigated by numerous authors. Initial neutron diffraction measurements indicated a collinear $1 \mathrm{k} \mathrm{AFM}^{15,16}$ ground-state, coupled with a homogeneous lattice distortion. ${ }^{19}$ Later studies suggested an internal Jahn-Teller distortion ${ }^{66,71-74}$ of the $\mathrm{O}^{2-}$ ions, but no evidence has been found for a reduction in the external cubic crystal symmetry. ${ }^{69,71,74,75}$ On further investigation, noncollinear $2 \mathrm{k}$ AFM order was proposed due to internal crystallographic distortion. ${ }^{71,74}$

Finally, low-temperature $(T<30.8 \mathrm{~K})$ neutron diffraction measurements of $\mathrm{UO}_{2}$ have confirmed an internal $\mathrm{Pa} \overline{3}$ (No. 205) crystallographic distortion, where the displacement of the $\mathrm{O}^{2-}$ ions is $0.014 \AA{ }^{13,20,71,74}$ which is indicative of transverse $3 \mathrm{k}$
AFM order. ${ }^{13,33,76}$ Anti-ferroquadrupolar ordering favours $\mathrm{Pa} \overline{3}$ (No. 205) crystal symmetry by minimizing quadrupolar and exchange terms. ${ }^{18}$ Neutron diffraction measurements have determined an ordered effective magnetic moment of $1.74 \mu_{\mathrm{B}}$ per $\mathrm{U}$ ion, ${ }^{71}$ whereas the transverse $3 \mathrm{k}$ AFM ground-state has been inferred by inelastic neutron scattering (INS), ${ }^{77}$ resonant $\mathrm{X}$-ray scattering $(\mathrm{RXS})^{72}$ and nuclear magnetic resonance $(\mathrm{NMR})^{20}$ measurements. In terms of the electronic structure, the f-f MottHubbard insulating character of $\mathrm{UO}_{2}$ with a band-gap of 2.00$2.50 \mathrm{eV}^{2,78,79}$ has been established by optical adsorption, ${ }^{2,79} \mathrm{X}$-ray adsorption (XAS), ${ }^{3,78,80}$ X-ray photoemission (XPS), ${ }^{81-84}$ bremsstrahlung isochromatic spectroscopy (BIS), ${ }^{83,84}$ resonant photoemission spectroscopy (RPES), ${ }^{85}$ inverse photoemission spectroscopy (IPES) ${ }^{86}$ and theoretical methods. ${ }^{48,87,88}$ In the past, multiple investigations of $\mathrm{UO}_{2}$ have focused on the $F m \overline{3} m$ (No. 225) crystal structure ${ }^{11,18,69,80,89,90}$ but in this study, in addition to the experimental AFM ground-state, the energetics of the DM and FM configurations have been considered for comparison with previous theoretical considerations. ${ }^{91,92}$

By comparison, the magnetic ground-state of $\mathrm{NpO}_{2}$ is highly complex and marred by several inconsistencies. ${ }^{22}$ In crystal field theory, the $\mathrm{Np}^{4+}$ ion results in the ${ }^{4} \mathrm{I}_{9 / 2} \quad\left(\Gamma_{8}\right.$ quartet $)$ configuration. Indeed, the tetravalent $\mathrm{f}^{3}$ nature of the $\mathrm{Np}^{4+}$ ion has been confirmed by Mossbauer isomer shift spectroscopy. ${ }^{93}$ At low-temperature $(T=25.4 \mathrm{~K})$, a first-order PM-AFM phase transition has been indicated by magnetic susceptibility ${ }^{94}$ and specific heat capacity measurements. ${ }^{95,96}$ In addition, a longitudinal 3k AFM ground-state coupled to $F m \overline{3} m$ (No. 225) crystal symmetry has been inferred from resonant X-ray scattering ${ }^{73}$ $(10 \mathrm{~K}<T<17 \mathrm{~K})$ and ${ }^{17} \mathrm{O}$ NMR measurements $(T=17 \mathrm{~K}) .{ }^{97}$ The absence of an external distortion of the cubic cell further indicates noncollinear 3k AFM behavior. ${ }^{96}$ In contrast, lowtemperature Mossbauer $(T=1.5 \mathrm{~K})^{93}$ and neutron diffraction $(12 \mathrm{~K}<T<30 \mathrm{~K})^{98}$ measurements have failed to identify a magnetic moment. The upper limit for the magnetic moment set by muon spin rotation measurements $(0.3 \mathrm{~K}<T<25.4 \mathrm{~K})$ is $0.06-0.15 \mu_{\mathrm{B}}$ per $\mathrm{Np}$ ion, ${ }^{22,99}$ whereas the upper limit for the magnetic moment set by Mössbauer spectroscopy is $0.01 \mu_{\mathrm{B}}$ per $\mathrm{Np}$ ion..$^{93}$ The absence of a measurable local magnetic moment is inconsistent with the idea of magnetic order and the nature of 
the small-moment AFM state is unresolved. ${ }^{22}$ As a Kramers ion, i.e. an ion with an uneven number of valence electrons, the ground $\mathrm{Np}^{4+}$ state should order magnetically in the absence of interactions that break time-reversal symmetry conditions. A mechanism by which the magnetic moment of the $\mathrm{Np}^{4+}$ ion can be suppressed is AFM super-exchange, ${ }^{22}$ which is the coupling between moment-bearing cations via nominally DM anions. ${ }^{100,101}$

The inhomogeneous nature of $\mathrm{NpO}_{2}$ samples has hindered experimental investigation. ${ }^{96,102}$ For instance, the extreme difficulty in manufacturing large single-crystal samples impacts the search for low-temperature $(T<25.4 \mathrm{~K})$ crystallographic distortions. ${ }^{96,103}$ In the past, the detection threshold of neutron diffraction measurements was limited to $0.02-0.03 \AA^{22}$ By comparison, crystallographic distortions of isostructural $\mathrm{UO}_{2}$ are of the order of 0.01-0.02 $\AA^{19}$ In a search for low-temperature $(T<25.4 \mathrm{~K})$ anharmonic effects in $\mathrm{NpO}_{2}$ by neutron diffraction $(12 \mathrm{~K}<$ $T<30 \mathrm{~K})^{98}$ and in an independent RXS $(9 \mathrm{~K}<T<25 \mathrm{~K})$ study, ${ }^{96}$ no evidence of a dynamical distortion of the $\mathrm{O}^{2-}$ ions was found. However, Mossbauer spectroscopy $(T=1.5 \mathrm{~K})$ of $\mathrm{NpO}_{2}$ has indicated an internal $\mathrm{O}^{2-}$ ion distortion inferred by the small broadening of spectroscopic lines. ${ }^{93}$ In addition, inelastic neutron scattering (INS) $\left(5 \mathrm{~K}<T<25 \mathrm{~K}\right.$ ) studies ${ }^{104,105}$ indicate an internal $\mathrm{O}^{2-}$ ion distortion of $0.02 \AA$, which is reminiscent of the internal $\mathrm{O}^{2-}$ ion distortion in $\mathrm{UO}_{2}$. The internal $\mathrm{O}^{2-}$ ion distortion may result in $\mathrm{Pa} \overline{3}$ (No. 205) crystal symmetry that is indicative of transverse $3 \mathrm{k}$ AFM behaviour, although this has yet to be experimentally confirmed.

The upper-limit of the magnetic moment (0.01-0.15 $\mu_{\mathrm{B}}$ per $\mathrm{Np}$ ion) indicates that $\mathrm{NpO}_{2}$ is a small-moment system. ${ }^{22,93,96,99}$ To our knowledge, small moments have only been identified in heavy-fermion metals ${ }^{106-108}$ and has yet to be identified in insulators. In this regard, computational investigations of $\mathrm{PuO}_{2}$ have indicated the existence of an unconfirmed small-moment insulating magnetic ground-state. ${ }^{1}$ For instance, multiple DFT calculations have indicated an AFM ground-state, which contrasts with the DM state established by experimental methods. ${ }^{109-112}$ The mechanisms behind such an intriguing electronic states are not yet fully understood; where, the crystal and magnetic structures of $\mathrm{NpO}_{2}$ remain unresolved.

\section{Computational methodology}

A noncollinear relativistic computational study of the $\mathrm{AnO}_{2}$ $(\mathrm{An}=\mathrm{U}, \mathrm{Np})$ magnetic structure by the Vienna $\mathrm{Ab}$ initio Simulation Package (VASP) code has been conducted. ${ }^{35,39,113}$ The investigation considers: hybrid Heyd-Scuseria-Ernzerhof (HSE06), ${ }^{40-42}$ PBEsol $+U,{ }^{36-38}$ and PBEsol ${ }^{31,32}$ functionals. ${ }^{31,32,36-38}$ A planewave basis set with a kinetic energy cut-off of $500 \mathrm{eV}$ has been used. The following valence electrons have been considered for: uranium $\left(6 s^{2}, 7 s^{2}, 6 p^{6}, 6 d^{2} 5 f^{2}\right)$, neptunium $\left(6 s^{2}, 7 s^{2}, 6 p^{6}\right.$, $\left.6 d^{2} 5 f^{3}\right)$, and oxygen $\left(2 s^{2}, 2 p^{4}\right)$. The influence of noncollinear magnetic behaviour and $\mathrm{SOI}^{14}$ has also been included. The spin quantisation axis is defined by $(0,0,1)$ plane, from which magnetic and spinor-like values are calculated. An ionic relaxation with the conjugate gradient algorithm has been completed. ${ }^{114}$
The space group has been evaluated to $10^{-5} \AA$ based on a symmetry analysis of the unit cell. Images are visualized by the VESTA code. ${ }^{115}$

As a computationally intensive method, hybrid functionals incorporate Hartree-Fock (HF) exchange energy into the DFT formulism. In this study, the hybrid HSE06 functional has been used. ${ }^{40-42,116}$ The integration of the Brillouin zone has been calculated from a $\Gamma$-centred $(4 \cdot 4 \cdot 4) k$-point grid with the conventional Gaussian method. ${ }^{117}$

$$
E_{\mathrm{XC}}^{\mathrm{HSE}}=(a) E_{\mathrm{X}}^{\mathrm{HF}, \mathrm{SR}}(\mu)+(1-a) E_{\mathrm{X}}^{\mathrm{PBE}, \mathrm{SR}}(\mu)+E_{\mathrm{X}}^{\mathrm{PBE}, \mathrm{LR}}(\mu)+E_{\mathrm{C}}^{\mathrm{PBE}}
$$

The terms define the exchange-correlation HSE06 energy $\left(E_{\mathrm{XC}}^{\mathrm{HSE}}\right)$, an adjustable constant $(a)$, the short-range $(\mathrm{SR})$ interaction energy and the long-range (LR) interaction energy. The adjustable screening $(\mu)$ modifier in the HSE06 functional is $0.207 \AA^{-1}$. Calculations were performed until self-consistency was reached for the electronic and ionic thresholds of $1 \times$ $10^{-6} \mathrm{eV}$ and $1 \times 10^{-2} \mathrm{eV} \mathrm{A}^{-1}$, respectively.

To capture the highly-correlated nature of f-electron compounds, DFT $+U$ offers a more computationally tractable method. ${ }^{31,32,36-38}$ The on-site Coulomb repulsion of the An 5 -electrons has been treated by the rotationally invariant Liechtenstein et al. $\mathrm{DFT}+U^{31,32,36-38}$ formulism, ${ }^{37}$ where the Coulomb $(U)$ and exchange $(J)$ modifiers are treated as independent variables:

$$
E_{\mathrm{dc}}(\hat{n})=\frac{U}{2} \hat{n}_{\mathrm{tot}}\left(\hat{n}_{\mathrm{tot}}-1\right) \frac{J}{2} \sum_{\sigma} \hat{n}_{\text {tot }}^{\sigma}\left(\hat{n}_{\text {tot }}^{\sigma}-1\right)
$$

The double counting energy term $\left(E_{\mathrm{dc}}\right)$, the on-site occupancy matrix $(n)$ and the spin index $(\sigma)$ are denoted by the terms in parentheses. Note: the Dudarev et $a l^{36}$ formulism and Liechtenstein et $a l^{37}$ formulism when $J=0.00 \mathrm{eV}$ are identical. ${ }^{118}$ The performance of numerous exchange-correlation functionals and $J$ on noncollinear magnetic materials has been investigated. ${ }^{33,118}$ The anisotropic nature of the f-states has been shown to increases with $J$ (and with $U$ ), and therefore $J$ is ignored in this study. ${ }^{33,48,118}$

In an earlier study on the $\mathrm{AnO}_{2}$, the performance of multiple DFT functionals was tested; ${ }^{33}$ where, the PBEsol functional had been proven to be the most effective. The exchange-correlation energy is therefore evaluated by the PBEsol functional. ${ }^{119}$ The integration of the Brillouin zone is performed with a $\Gamma$-centred $(5 \cdot 5 \cdot 5) k$-point grid, ${ }^{117}$ using the Blöchl tetrahedron method. ${ }^{120}$ Self-consistent calculations were performed until convergence was reached for the respective electronic and ionic thresholds of $1 \times 10^{-8} \mathrm{eV}$ and $1 \times 10^{-3} \mathrm{eV} \mathrm{A}^{-1}$. For the optical absorbance calculation, the $k$-point mesh is $15 \cdot 15 \cdot 15$; for band structure calculations, the Fmmm (No. 69) $k$-point pathway is $\Gamma \rightarrow Y \rightarrow$ $X \rightarrow Z \rightarrow \Gamma \rightarrow L$, whereas, the $P a \overline{3}$ (No. 205) $k$-point pathway is $\Gamma \rightarrow M \rightarrow R \rightarrow X \rightarrow \Gamma \rightarrow R$.

\section{Results \& discussions}

\subsection{Uranium dioxide}

3.1.1 Magnetic structure. The electronic and crystallographic nature of $\mathrm{UO}_{2}$ is influenced by the magnetic state. An energetically 
Table 2 The relative energy (eV), band-gap (eV), magnetic moment ( $\mu_{\mathrm{B}}$ per $\mathrm{U}$ ion), lattice volume $\left(\AA^{3}\right)$ and space group (number) for each $\cup \mathrm{O}_{2}$ magnetic configuration. Calculated by the HSE06 functional. The energetics of the magnetic configurations are calculated relative to the degenerate transverse $1-$ $2 \mathrm{k}$ antiferromagnetic ground-state

\begin{tabular}{|c|c|c|c|c|c|c|}
\hline Initial configuration & & Relative energy (eV) & Band-gap (eV) & Magnetic moment ( $\mu_{\mathrm{B}}$ per $\mathrm{U}$ ion) & Lattice volume $\left(\AA^{3}\right)$ & Space group (number) \\
\hline \multicolumn{7}{|l|}{ Diamagnetic } \\
\hline & & 0.659 & 1.75 & 0.85 & 161.70 & $F m \overline{3} m$ (No. 225) \\
\hline \multicolumn{7}{|l|}{ Ferromagnetic } \\
\hline & (001) & 0.010 & 2.42 & 1.53 & 162.78 & I4/mmm (No. 139) \\
\hline & $(011)$ & 0.026 & 2.62 & 1.45 & 162.81 & Immm (No. 71) \\
\hline & (111) & 0.012 & 2.57 & 1.44 & 162.60 & $R \overline{3} m$ (No. 166) \\
\hline \multicolumn{7}{|l|}{ Antiferromagnetic } \\
\hline \multirow{3}{*}{ Longitudinal } & $1 \mathrm{k}$ & 0.006 & 2.79 & 1.51 & 162.63 & I4/mmm (No. 139) \\
\hline & $2 \mathrm{k}$ & 0.019 & 2.88 & 1.43 & 162.48 & $I 4 / \mathrm{mmm}$ (No. 139) \\
\hline & $3 \mathrm{k}$ & 0.023 & 2.82 & 1.42 & 162.47 & $F m \overline{3} m$ (No. 225$)$ \\
\hline \multirow[t]{3}{*}{ Transverse } & $1 \mathrm{k}$ & 0.000 & 3.02 & 1.49 & 162.53 & Fmmm (No. 69) \\
\hline & $2 \mathrm{k}$ & 0.000 & 3.02 & 1.49 & 162.55 & $P b c a$ (No. 61) \\
\hline & $3 \mathrm{k}$ & 0.014 & 2.99 & 1.43 & 162.57 & $P a \overline{3}$ (No. 205) \\
\hline \multicolumn{2}{|l|}{ Experimental } & - & $2.00-2.50^{79,121}$ & $1.74^{69,71,74}$ & $163.85^{69,74}$ & $P a \overline{3}(\text { No. } 205)^{13}$ \\
\hline
\end{tabular}

Note: the initial DM HSE06 state is unstable; a relaxed, highly-energetic FM (852) state has been identified.

degenerate transverse 1-2k AFM HSE06 ground-state has been calculated (Table 2). In addition, a metastable, highly-energetic, weakly FM (852) configuration ( $0.85 \mu_{\mathrm{B}}$ per U ion) from the initial DM HSE06 state has been identified. A comparatively insignificant low-index FM and AFM state (1.42-1.53 $\mu_{\mathrm{B}}$ per U ion) energy difference has been found. In relation to the experimental transverse 3k AFM ground-state, the FM (001), FM (111), longitudinal 1k AFM and transverse 1-2k AFM states are marginally lower in energy by $-0.01 \mathrm{eV}$-formula unit $^{-1}$. A band-gap of $2.42-2.57 \mathrm{eV}$ (for the low-index FM states) and 2.82-3.02 eV (for the AFM states) has been calculated, which is considerably higher when contrasted against experimental measurements. It is noted that the transverse $1 \mathrm{k}$ AFM state is consistent with experimental static and lowfrequency dynamical magnetic measurements. ${ }^{22}$ A crystallographic Fmmm (No. 69) or Pbca (No. 61) distortion in the transverse 1-2k AFM states has been found, ${ }^{13}$ whereas the crystallographic $\mathrm{Pa} \overline{3}$ (No. 205) distortion of the transverse 3k AFM state agrees with neutron diffraction measurements. ${ }^{13,20,71,74}$

To reduce the computational cost and to further probe the magnetic ground-state, the relative energetics of competing magnetic phases have been calculated with PBEsol+ $U$. The relative energetics of the magnetic states are influenced by the U modifier (Fig. 3). In contrast to hybrid HSEE06 calculations, the highly-energetic PBEsol $+U$ DM state is stable. In our calculations, the metallic FM (111) ground-state, calculated by PBEsol $(U=0$ $\mathrm{eV}$ ), is inconsistent with the experimental data. Indeed, under no circumstance is the insulating nature of $\mathrm{UO}_{2}$ reproduced when $U=0 \mathrm{eV}$, which illustrates the failure of pure DFT to account for the highly localized character of the 5 f electrons. The insulating nature of $\mathrm{UO}_{2}$, which is well-described experimentally, can be reproduced computationally when $U=3-4 \mathrm{eV}$. The introduction of the $U$ modifier immediately results in an AFM ground-state. The nature of the AFM ground-state is, however, dependent on the $U$ constraint. A degenerate longitudinal 2-3k AFM and transverse 1-3k AFM ground-state is formed when $U=1-7 \mathrm{eV}$. In comparison with HSE06 calculations, the relative degeneracy of the transverse 1-2k AFM PBEsol+ $U$ ground-states has also been shown. In relation to past theory, the DM is considerably higher in energy and therefore physically unrealistic.

The low-temperature crystal structure of $\mathrm{UO}_{2}$ shows $\mathrm{Pa} \overline{3}$ crystal symmetry indicated by neutron scattering and X-ray diffraction measurements. ${ }^{13}$ In our calculations, the transverse 3k AFM state results in cubic $P a \overline{3}$ (No. 205) crystal symmetry, and is consistent with experimental information. ${ }^{13}$ As a model of the cubic crystal structure at low-temperature, the collinear $1 \mathrm{k}$ AFM states are an invalid approximation. The transverse $1 \mathrm{k}$ AFM with orthorhombic Fmmm (No. 69) symmetry differs from the longitudinal 1k AFM state with tetragonal $14 / \mathrm{mmm}$ (No. 139) symmetry. The magnetic moment of $\mathrm{UO}_{2}$ is $1.74 \mu_{\mathrm{B}}$ per $\mathrm{U}$ ion, reported by neutron diffraction measurements. ${ }^{69,71,74}$ In our calculations, the magnetic moment of the $U$ ion is underestimated by DFT $+U$ and the hybrid HSE06 functional with the closest approximation obtained by the FM (111) states. In terms of the lattice volume, no discernible change is detected between the ordered magnetic states, but the lattice volume is considerably lower in the DM state.

3.1.2 Electronic structure. The electronic structure of the transverse $3 \mathrm{k}$ AFM state for $\mathrm{UO}_{2}$ has previously been calculated by first-principles methods $(U=3.35 \mathrm{eV}, J=0.00 \mathrm{eV}) ;^{33}$ however, a degenerate transverse 1-2k AFM ground-state has been identified by HSE06 and PBEsol $+U$ calculations. As experimental static low-frequency dynamical magnetic measurements ${ }^{22}$ and initial neutron diffraction ${ }^{15,16}$ studies found a transverse $1 \mathrm{k}$ AFM ground-state, the electronic structure of the transverse $1 \mathrm{k}$ AFM ground-state has been calculate by $\operatorname{PBEsol}+U(U=3.50 \mathrm{eV}$, $J=0.00 \mathrm{eV}$ ). The modifier is consistent with other studies within the literature and mirrors the experimental band-gap information (Fig. 4). In contrast, collinear $1 \mathrm{k}$ AFM calculations by constrained random phase approximation (cRPA) methods were obtained with $U=5.70 \mathrm{eV}$ and $J=0.40 \mathrm{eV},{ }^{122}$ i.e. considerably higher than in the literature. ${ }^{48,83,90,123,124}$

In the density of states (DoS), the valence band maximum (VBM) and conduction band minimum (CBM) are mainly comprised of uranium f-states. The $U$ d-states are significantly higher in 

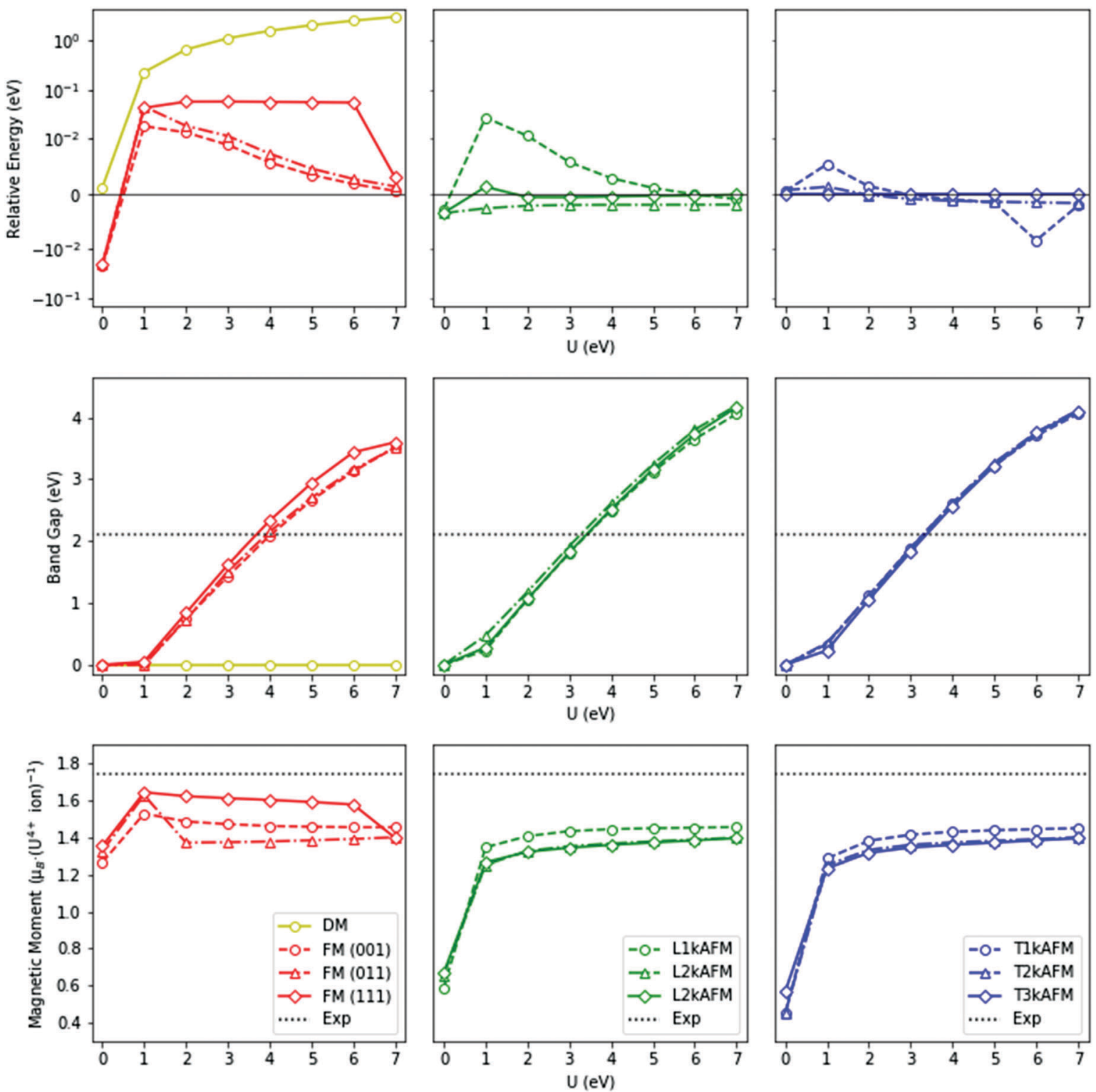

Fig. 3 The relative ground-state energies, band-gaps, and effective magnetic moments against the Coulomb modifier (U) for diamagnetic (DM), ferromagnetic (FM), and antiferromagnetic (AFM) states of $\mathrm{UO}_{2}$, calculated with PBEsol $+U$. The antiferromagnetic transverse (T) and longitudinal ( $L$ ) domains are also represented. The k-prefix denotes the number of independent wave vectors: above, the calculated energy of magnetic states relative to the transverse 3k antiferromagnetic state; middle, the fundamental band-gap; below, the effective magnetic moment of the uranium ions. The DM (yellow), FM (orange), longitudinal AFM (green) and transverse AFM (blue) states are indicated.
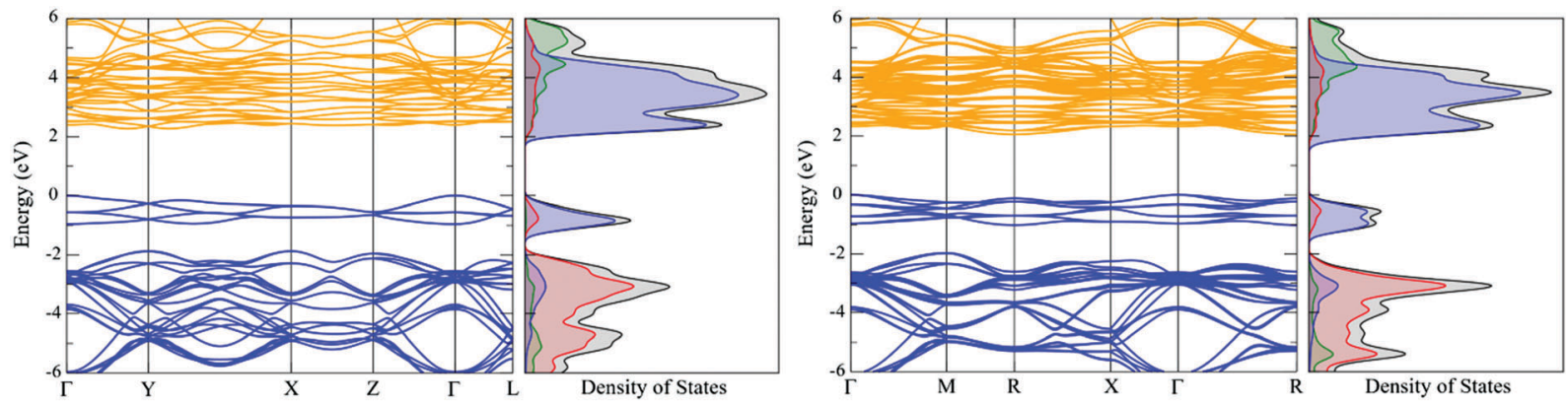

Fig. 4 The electronic structure of $\mathrm{UO}_{2}$ calculated by PBEsol for the: (left) transverse $1 \mathrm{k}$ AFM state $(U=3.50 \mathrm{eV}, \mathrm{J}=0.00 \mathrm{eV}$ ); (right) transverse $3 \mathrm{k}$ AFM state $(U=3.35 \mathrm{eV}, \mathrm{J}=0.00 \mathrm{eV}$ ). The valence band (dark blue), conduction band (orange), $\mathrm{U} \mathrm{f}-$ (blue), $\mathrm{U} \mathrm{d}$ - (green) and $\mathrm{O} \mathrm{p}$ - (red) states are indicated. 
Table 3 The fundamental band-gap (eV), optical band-gap (eV), lattice constant ( $\AA$ ), bulk modulus (GPa) and magnetic moment ( $\mu_{\mathrm{B}}$ per $\mathrm{U}$ ion) for the transverse $3 \mathrm{k}(\mathrm{T}-3 \mathrm{k}) \mathrm{AFM}$ state of $\mathrm{UO}_{2}$

\begin{tabular}{|c|c|c|c|c|c|c|c|c|}
\hline \multicolumn{2}{|l|}{$\mathrm{DFT}+U$} & \multicolumn{2}{|l|}{ Band-gap (eV) } & \multirow{2}{*}{$\begin{array}{l}\text { Lattice } \\
\text { constant }(\AA)\end{array}$} & \multirow{2}{*}{$\begin{array}{l}\text { Bulk } \\
\text { modulus (GPa) }\end{array}$} & \multirow{2}{*}{$\begin{array}{l}\text { Magnetic } \\
\text { moment ( } \mu_{\mathrm{B}} \text { per } \mathrm{U} \text { ion) }\end{array}$} & \multirow[b]{2}{*}{ Crystal symmetry } & \multirow[b]{2}{*}{ Magnetic state } \\
\hline$U(\mathrm{eV})$ & $J(\mathrm{eV})$ & Fundamental & Optical & & & & & \\
\hline 3.50 & 0.00 & 2.27 & 2.49 & 5.476 & 210 & 1.42 & Fmmm (69) & T-1k AFM \\
\hline 3.35 & 0.00 & 2.06 & 2.20 & 5.474 & 210 & 1.35 & $P a \overline{3}(205)$ & T-3k AFM ${ }^{33}$ \\
\hline- & - & $2.00-2.50^{79,121}$ & & $\sim 5.473^{69,74}$ & $207^{11}$ & $1.74^{69,71,74}$ & $\mathrm{~Pa} \overline{3}$ (205) & Experimental \\
\hline
\end{tabular}

energy and should therefore have very little influence on bonding interactions. This electronic structure indicates that $\mathrm{UO}_{2}$ is a MottHubbard insulator, consistent with experimental information. The band structure of transverse $1 \mathrm{k} \mathrm{AFM} \mathrm{UO}_{2}$ ground-state results in a $\Gamma$ (VBM) to $\Gamma-Y$ (CBM) indirect fundamental band-gap of $2.27 \mathrm{eV}$; whereas the calculated optical absorption spectra band-gap is $2.49 \mathrm{eV}$. The fundamental band-gap and optical band-gap (although they are not strictly directly comparable) differ by $0.22 \mathrm{eV}$. The fundamental band-gap defines the VBM-CBM energy difference, whereas, the optical band-gap defines the minimum allowed transition as controlled by symmetry rules. The calculated band-gap and bulk modulus are in very good agreement with experimental information (Table 3). The electronic structure of the transverse $3 \mathrm{k}$ AFM is also shown; here, the degeneracy of the bands is noticeably perturbed by noncollinear order.

\subsection{Neptunium dioxide}

3.2.1 Magnetic structure. In this section we have calculated the relative energetics for each magnetic state, the band-gap, the magnetic moment of the $\mathrm{Np}$ ion, and the crystal structure for $\mathrm{NpO}_{2}$. Note: as of the $\mathrm{f}^{3}$ nature of the $\mathrm{Np}^{4+}$ Kramers ion, the DM configuration is physically unrealistic. In our hybrid functional HSE06 calculations, the transverse 3k AFM state is only $0.002 \mathrm{eV}$.formula unit ${ }^{-1}$ higher in energy than the FM (111) ground-state (Table 4). The transverse 3k AFM state results in $P a \overline{3}$ (No. 205) crystal symmetry, which satisfies observations of noncollinear magnetic behaviour and the inferred internal crystallographic distortion. However, the magnetic moment is anomalously high and does not fit the picture of a small-moment AFM state. As with $\mathrm{UO}_{2}$ and $\mathrm{PuO}_{2},{ }^{1}$ the crystal symmetry and magnetic structure of $\mathrm{NpO}_{2}$ are coupled by SOI. The low temperature crystal structure of $\mathrm{NpO}_{2}$ is unresolved, but thus far, $P a \overline{3}$ (No. 205) and $P n \overline{3} m$ (No. 224) structures have been inferred from RXS measurements. ${ }^{125}$

The HSE06 results above are emulated by PBEsol $+U$ (Fig. 5). The FM (111) ground-state, calculated by PBEsol, independent of the $U$ modifier, results in $R \overline{3} m$ (No. 166) symmetry as a result of a trigonal distortion of the unit cell. However, this structure is inconsistent with current experimental data. A number of experimental investigations have strongly indicated an AFM ground-state, although the nature of the magnetic moment has thus far not been determined. No evidence of a trigonal $R \overline{3} m$ (No. 166) crystallographic distortion has been reported, but noncollinear 3k AFM behaviour has been identified, where the domain is unresolved..$^{96,97,105,125,128}$ From our calculations, only the noncollinear 3k AFM states result in cubic symmetry. The transverse 3k AFM state is marginally lower in energy than the longitudinal 3k AFM state (Table 4), with the external cubic symmetry retained in both cases.

The electronic structure of $\mathrm{NpO}_{2}$ is influenced by the magnetic state. The measured optical absorbance band-gap of $\mathrm{NpO}_{2}$ epitaxial thin films is $2.85 \mathrm{eV} .{ }^{126} \mathrm{In}$ our calculations, the correct insulating nature of $\mathrm{NpO}_{2}$ is reproduced when $U=4-6 \mathrm{eV}$ for all magnetic states. In this range, the relative energy differences between the longitudinal 3k AFM, transverse 2-3k AFM, and the FM (111) states are minimal.

The longitudinal 3k AFM state results in $F m \overline{3} m$ (No. 225) symmetry, which is supported by RXS measurements. In contrast, the transverse $3 \mathrm{k}$ AFM state results in $P a \overline{3}$ (No. 205) symmetry, characterized by an internal $\mathrm{O}^{2-}$ distortion of $0.011 \AA$. The distortion is equal to that implied by Mossbauer spectroscopy ${ }^{93}$ and INS ${ }^{104,105}$ measurements. In theory, the noncollinear AFM

Table 4 The relative energy (eV), band-gap (eV), magnetic moment ( $\mu_{\mathrm{B}}$ per $\mathrm{Np}$ ion), lattice volume $\left(\AA^{3}\right)$ and space group (number) for each $\mathrm{NpO} \mathrm{O}_{2}$ magnetic configuration, calculated by the HSEO6 functional. The energetics of the magnetic configurations are calculated relative to the ferromagnetic (111) ground-state

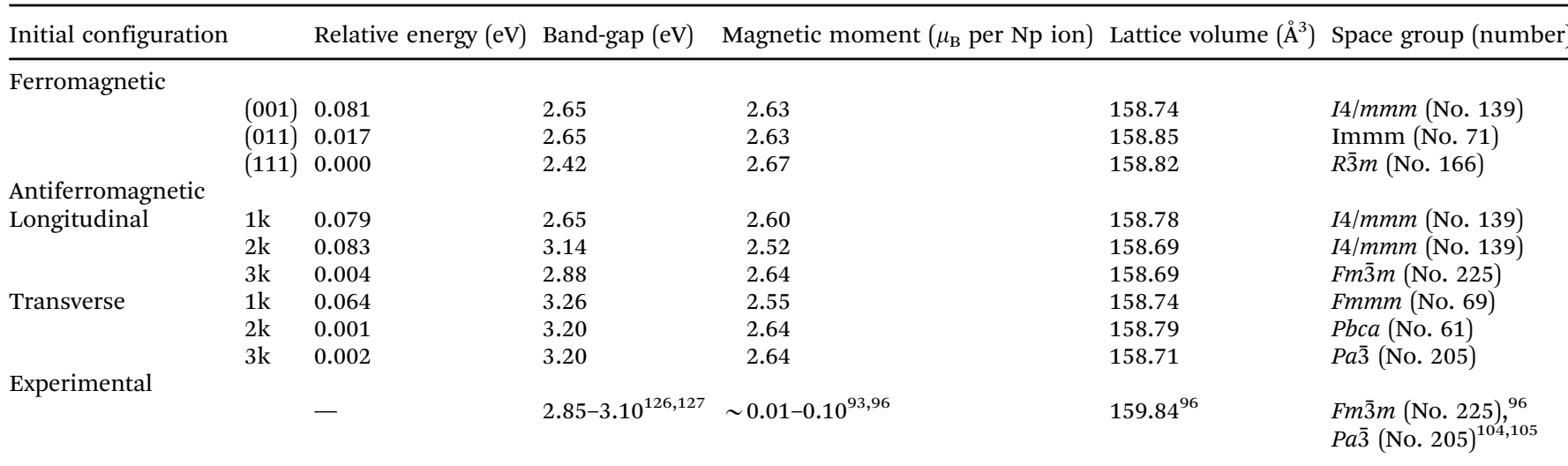



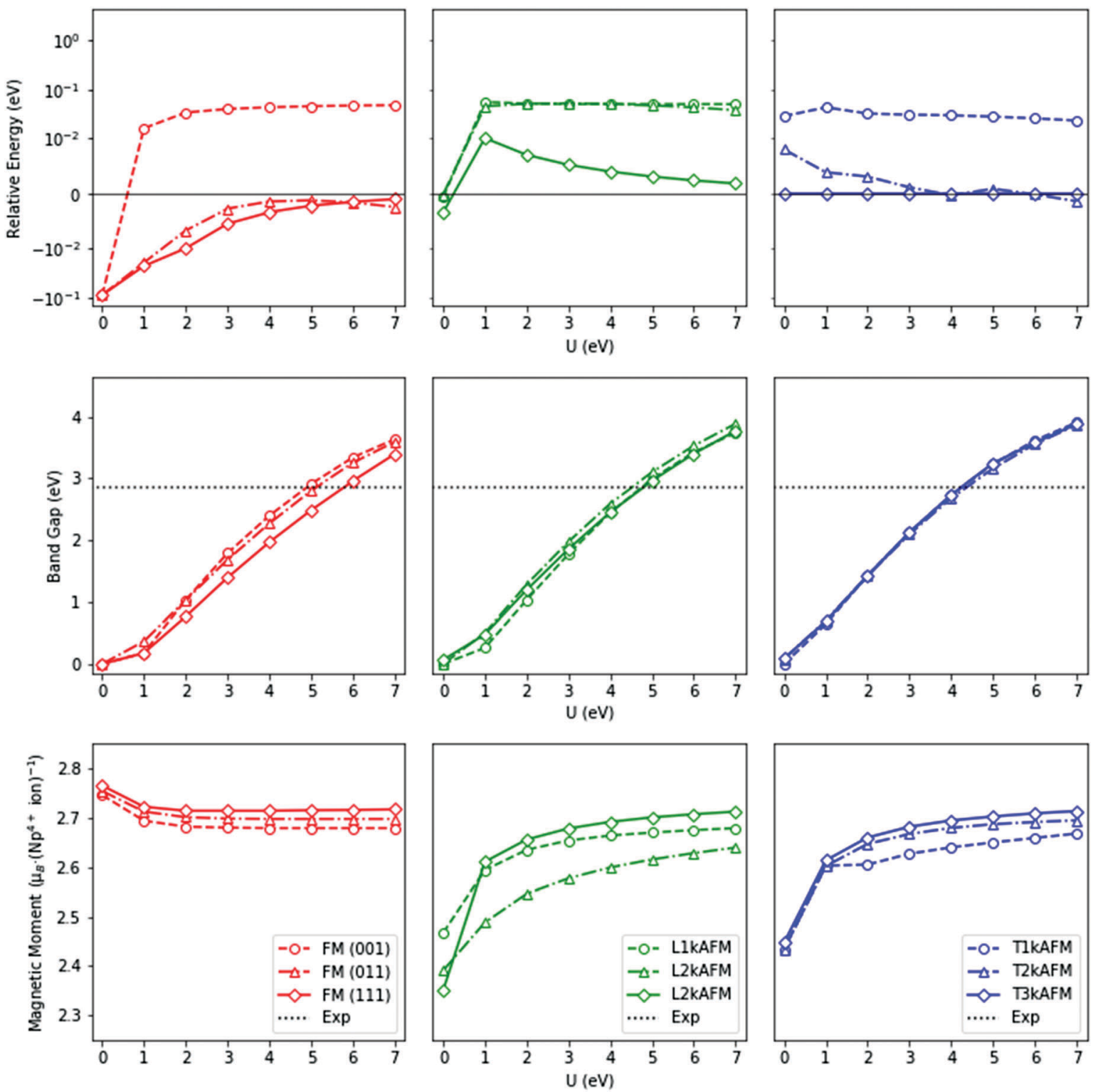

Fig. 5 The relative ground-state energies, band-gaps, and effective magnetic moments against the Coulomb modifier (U) for ferromagnetic (FM), and antiferromagnetic (AFM) states of $\mathrm{NpO}_{2}$, calculated with PBEsol+U. The antiferromagnetic transverse (T) and longitudinal (L) domains are additionally represented. The k-prefix denotes the number of independent wave vectors: above, the calculated energy of magnetic states relative to the transverse $3 \mathrm{k}$ antiferromagnetic state; middle, the fundamental band-gap; below, the effective magnetic moment of the neptunium ions. The FM (orange), longitudinal AFM (green) and transverse AFM (blue) states are indicated.

domain can be established by its crystalline environment, but the distortion cannot be confirmed as this is below the instrument resolution. In terms of the lattice volume, no discernible change between different magnetic states is observed.

As mentioned above, the magnetic moment of the Np ions is unresolved. Experimentally, the $\mathrm{NpO}_{2}$ system appears to be a small-moment system, but this picture cannot be confirmed by first-principle methods. The magnetic moment of the Np ions in the FM states decreases from $2.77 \mu_{\mathrm{B}}$ per Np ion to $2.68 \mu_{\mathrm{B}}$ per Np ion when $U$ ranges from $0-7 \mathrm{eV}$, whereas in AFM states, it increases from $2.35 \mu_{\mathrm{B}}$ per Np ion to $2.71 \mu_{\mathrm{B}}$ per Np ion for the same values of $U=0-7 \mathrm{eV}$. In contrast with low-temperature experimental measurements, the calculated magnetic moment is considerably greater. A superexchange-type mechanism in $\mathrm{NpO}_{2}$ could result in a small-moment system, for which DFT-based methods have been shown to be unsuitable. ${ }^{19,22,129}$ It is noted that in reference to the high-temperature PM state, the calculated magnetic moment is in close agreement.

3.2.2 Electronic structure. No experimental information on $\mathrm{NpO}_{2}$ exists to support a FM (111) ground-state. The unopposed and overwhelming consensus is that $\mathrm{NpO}_{2}$ possesses an AFM ground-state. ${ }^{96,98,105,128,130}$ The influence of entropy on the lowtemperature magnetic state is unknown, which is believed to impact the magnetic ground-state. In addition, the influence of phonon activity on dynamic stability has yet to be studied. The calculated transverse $3 \mathrm{k}$ AFM state is consistent with observation of: 

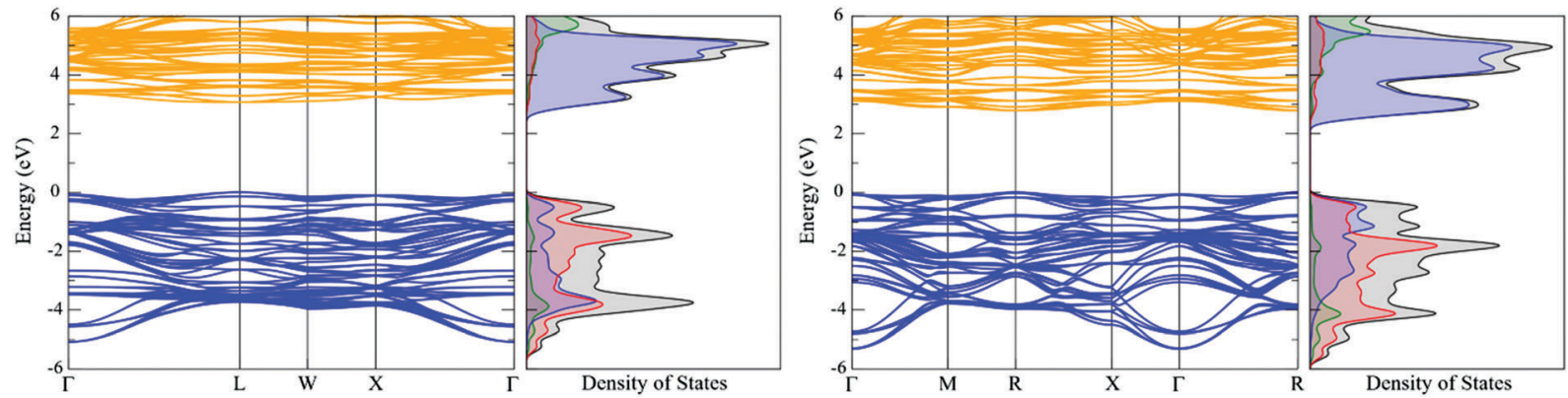

Fig. 6 The electronic structure of $\mathrm{NpO}_{2}$ calculated by PBEsol for the: (left) longitudinal 3k AFM state $(U=5.00 \mathrm{eV}, \mathrm{J}=0.75 \mathrm{eV})$; (right) transverse $3 \mathrm{k}$ AFM state $(U=4.25 \mathrm{eV}, J=0.00 \mathrm{eV}$ ). The valence band (dark blue), conduction band (orange), Np f- (blue), Np d- (green) and O p- (red) states are indicated.

Table 5 The fundamental band-gap (eV), optical band-gap (eV), lattice constant ( $\AA$ ), bulk modulus (GPa) and magnetic moment ( $\mu_{\mathrm{B}}$ per Np ion) for the longitudinal 3k (L-3K) AFM and transverse 3k (T-3k) AFM state of $\mathrm{NpO}_{2}$

\begin{tabular}{|c|c|c|c|c|c|c|c|c|}
\hline \multicolumn{2}{|l|}{$\mathrm{DFT}+U$} & \multicolumn{2}{|l|}{ Band-gap (eV) } & \multirow{2}{*}{$\begin{array}{l}\text { Lattice } \\
\text { constant }(\AA)\end{array}$} & \multirow{2}{*}{$\begin{array}{l}\text { Bulk } \\
\text { modulus (GPa) }\end{array}$} & \multirow{2}{*}{$\begin{array}{l}\text { Magnetic moment } \\
\left(\mu_{\mathrm{B}} \text { per Np ion }\right)\end{array}$} & \multirow[b]{2}{*}{ Crystal symmetry } & \multirow[b]{2}{*}{ Magnetic state } \\
\hline$U(\mathrm{eV})$ & $J(\mathrm{eV})$ & Fundamental & Optical & & & & & \\
\hline 4.25 & 0.00 & 2.79 & 2.81 & 5.442 & 215 & 2.70 & $P a \overline{3}$ (205) & T-3k AFM \\
\hline- & - & $2.85-3.10^{126,127}$ & & $5.427^{96}$ & $200^{11}$ & $\sim 0.01-0.10^{93,96}$ & 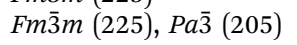 & Experimental \\
\hline
\end{tabular}

external cubic symmetry, and noncollinear magnetic behaviour. In addition, the inferred internal $\mathrm{O}^{2-}$ distortion is identical to observations of $\mathrm{Pa} \overline{3}$ crystal symmetry in $\mathrm{UO}_{2} \cdot{ }^{13}$ We therefore employed the same approach used for $\mathrm{UO}_{2}$, whereby the electronic structure of the transverse $3 \mathrm{k} \operatorname{AFM}(U=4.25 \mathrm{eV})$ has been calculated by the PBEsol functional (Fig. 6). The electronic structure of the longitudinal 3k AFM state with $F m \overline{3} m$ crystal symmetry has been shown for comparison; here, the $U(5.00 \mathrm{eV})$ and $J(0.75 \mathrm{eV})$ values are taken from an earlier investigation. ${ }^{33,48}$

In the transverse $3 \mathrm{k}$ AFM state, the CMB is formed equally of oxygen p-states and neptunium f-states, indicating that $\mathrm{NpO}_{2}$ shares both Mott and charge-transfer characteristics. In contrast, the longitudinal 3k AFM state predominately results in a charge-transfer insulator. ${ }^{33}$ The charge-transfer characteristics are likely due to the absence of an exchange modifier that otherwise serves to reduce the effective $\mathrm{Np}$ magnetic moment. In both instances, the $\mathrm{Np} d$-states have no significant role in chemical bonding interactions. The band structure reveals a direct $R$-centred band-gap of $2.79 \mathrm{eV}$, which compares with a calculated optical absorbance of $2.81 \mathrm{eV}$. Finally, the calculated bulk modulus of $\mathrm{NpO}_{2}$ for the longitudinal 3k AFM state is 214 $\mathrm{GPa}$, whereas the bulk modulus for the transverse $3 \mathrm{k}$ AFM state is $215 \mathrm{GPa}$ (Table 5).

\section{Conclusions}

The magnetic structure of $\mathrm{UO}_{2}$ and $\mathrm{NpO}_{2}$ has been investigated by first-principles methods. The influence of the magnetic structure on crystal symmetry is considered, and has been observed in other systems. ${ }^{1}$ The direction and magnitude of the ionic magnetic forces in the $\mathrm{AnO}_{2}$ introduce stresses within the crystal, which influences the low-temperature structure. In our calculations, the crystal structure and magnetic environment are closely interlinked. The experimental cubic environment is only preserved by noncollinear 3k AFM states. The longitudinal 3k AFM state results in $F m \overline{3} m$ (No. 225) crystal symmetry, whereas the energetically marginally more favourable transverse $3 \mathrm{k}$ AFM state results in $\mathrm{Pa} \overline{3}$ (No. 205) crystal symmetry, with a distortion of the $\mathrm{O}^{2-}$ ions of $0.011 \AA$. In contrast, the collinear $1 \mathrm{k}$ AFM states, often employed in past investigations, result in either an orthorhombic Fmmm (No. 69) or tetragonal $I 4 / \mathrm{mmm}$ (No. 139) distortion. ${ }^{131}$

A degenerate transverse 1-2k AFM ground-state for $\mathrm{UO}_{2}$ has been calculated, where the transverse $1 \mathrm{k}$ AFM state is in agreement with both static and low-frequency measurements of spin-wave excitations. ${ }^{22}$ The result contradicts the transverse 3k AFM state with $\mathrm{Pa} \overline{3}$ (No. 205) crystal symmetry found by: INS, ${ }^{77}$ RXS, and NMR measurements. ${ }^{20,77}$ As the ordered magnetic states are in close energetic proximity $\left(<0.026\right.$ eV F.U. $\left.{ }^{-1}\right)$, thermal fluctuations are thought to play a significant role and the influence of entropy on the dynamic stability remains unknown. In addition, a FM (111) $\mathrm{NpO}_{2}$ ground-state with $R \overline{3} m$ (No. 166) has been found. This contradicts resonant X-ray scattering ${ }^{73}$ and ${ }^{17} \mathrm{O}$ NMR measurements. ${ }^{97}$ In contrast, the transverse $3 \mathrm{k}$ AFM state with $P a \overline{3}$ (No. 205) crystal symmetry is only $0.001 \mathrm{eV}$ higher in energy. This magnetic structure has been linked to experimental measurements. ${ }^{96,98,105,128,130}$ The insulating nature of $\mathrm{NpO}_{2}$ is reproduced by $\mathrm{PBEsol}+U$ when $U=4-6 \mathrm{eV}$ for all magnetic configurations. In this study, the existence of a small-moment system cannot be confirmed. ${ }^{22,106,107}$

As the magnetic ground-sate from HSE06 calculations can be realised by PBEsol $+U$ (where an appropriate $U$ value has been chosen), PBEsol $+U$ offers a means of modelling the electronic structure in larger systems. ${ }^{132}$ To keep the methodology concurrent with the commonly used Dudarev et al. formulism, the choice of $U$ for transverse $1 \mathrm{k} \mathrm{AFM} \mathrm{UO} \mathrm{U}_{2}(3.50 \mathrm{eV})$ and transverse 
3k AFM $\mathrm{NpO}_{2}(4.25 \mathrm{eV})$ where $J=0.00 \mathrm{eV}$ has been shown. To avoid the crystallographic distortion of cubic symmetry found in the transverse $1 \mathrm{k} \mathrm{AFM} \mathrm{UO}_{2}$ state, transverse $3 \mathrm{k}$ AFM $(U=3.35 \mathrm{eV}, J=0.00 \mathrm{eV})$ order can also be used to emulate the electronic structure.

\section{Conflicts of interest}

There are no conflicts to declare.

\section{Acknowledgements}

This research was supported by the UK Engineering \& Physical Science Research Council (EPSRC) (Grant no. EP/G036675 and EP/K016288) and the Atomic Weapons Establishment (AWE). AES gratefully acknowledges the United States Department of Homeland Security (DHS), Domestic Nuclear Detection Office (DNDO), National Technical Nuclear Forensics Centre (NTNFC), for a Postdoctoral Research Fellowship. NHdL thanks AWE for a William Penney Fellowship. This work made use of the ARCHER UK National Supercomputing Service (http://www.archer.ac.uk), via our membership of the UK's HEC Materials Chemistry Consortium, which is funded by EPSRC (EP/L000202).

\section{Notes and references}

1 J. T. Pegg, A. E. Shields, M. T. Storr, A. S. Wills, D. O. Scanlon and N. H. de Leeuw, Phys. Chem. Chem. Phys., 2018, 20, 20943-20951.

2 J. Schoenes, Phys. Rep., 1980, 63, 301-336.

3 G. Kalkowski, G. Kaindl, W. D. Brewer and W. Krone, Phys. Rev. B: Condens. Matter Mater. Phys., 1987, 35, 2667-2677.

4 M. Noe and J. Fuger, Inorg. Nucl. Chem. Lett., 1974, 10, 7-19. 5 A. Arrott and J. E. Goldman, Phys. Rev., 1957, 108, 948-953. 6 J. K. Dawson and M. W. Lister, J. Chem. Soc., 1950, 2181-2187, DOI: 10.1039/JR9500002181.

7 C. E. McNeilly, J. Nucl. Mater., 1964, 11, 53-58.

8 T. N. Taylor and W. P. Ellis, Surf. Sci., 1981, 107, 249-262.

9 I. D. Prodan, G. E. Scuseria, J. A. Sordo, K. N. Kudin and R. L. Martin, J. Chem. Phys., 2005, 123, 014703.

10 Y. Tokunaga, H. Sakai, T. Fujimoto, S. Kambe, R. E. Walstedt, K. Ikushima, H. Yasuoka, D. Aoki, Y. Homma, Y. Haga, T. D. Matsuda, S. Ikeda, E. Yamamoto, A. Nakamura, Y. Shiokawa, K. Nakajima, Y. Arai and Y. Ōnuki, J. Alloys Compd., 2007, 444-445, 241-245.

11 M. Idiri, T. Le Bihan, S. Heathman and J. Rebizant, Phys. Rev. B: Condens. Matter Mater. Phys., 2004, 70, 014113.

12 S. D. Conradson, B. D. Begg, D. L. Clark, C. den Auwer, M. Ding, P. K. Dorhout, F. J. Espinosa-Faller, P. L. Gordon, R. G. Haire, N. J. Hess, R. F. Hess, D. Webster Keogh, G. H. Lander, D. Manara, L. A. Morales, M. P. Neu, P. PavietHartmann, J. Rebizant, V. V. Rondinella, W. Runde, C. Drew Tait, D. Kirk Veirs, P. M. Villella and F. Wastin, J. Solid State Chem., 2005, 178, 521-535.
13 L. Desgranges, Y. Ma, P. Garcia, G. Baldinozzi, D. Siméone and H. E. Fischer, Inorg. Chem., 2016, 56, 321-326.

14 S. Steiner, S. Khmelevskyi, M. Marsmann and G. Kresse, Phys. Rev. B: Condens. Matter Mater. Phys., 2016, 93, 224425.

15 S. J. Allen, Phys. Rev., 1968, 166, 530-539.

16 S. J. Allen, Phys. Rev., 1968, 167, 492-496.

17 V. S. Mironov, L. F. Chibotaru and A. Ceulemans, Advances in Quantum Chemistry, Academic Press, 2003, vol. 44, pp. 599-616.

18 P. Giannozzi and P. Erdös, J. Magn. Magn. Mater., 1987, 67, 75-87.

19 P. Santini, S. Carretta, G. Amoretti, R. Caciuffo, N. Magnani and G. H. Lander, Rev. Mod. Phys., 2009, 81, 807-863.

20 K. Ikushima, S. Tsutsui, Y. Haga, H. Yasuoka, R. E. Walstedt, N. M. Masaki, A. Nakamura, S. Nasu and Y. Ōnuki, Phys. Rev. B: Condens. Matter Mater. Phys., 2001, 63, 104404.

21 T. Sabine, G. Smith and K. Reeve, J. Phys. C: Solid State Phys., 1974, 7, 4513.

22 P. Santini, R. Lémanski and P. Erdős, Adv. Phys., 1999, 48, 537-653.

23 J. M. Haschke, J. Alloys Compd., 1998, 278, 149-160.

24 J. M. Haschke and T. H. Allen, J. Alloys Compd., 2001, 320, 58-71.

25 J. M. Haschke, T. H. Allen and J. C. Martz, J. Alloys Compd., 1998, 271-273, 211-215.

26 J. M. Haschke, T. H. Allen and L. A. Morales, J. Alloys Compd., 2001, 314, 78-91.

27 J. M. Haschke, T. H. Allen and L. A. Morales, Los Alamos Sci., 2000, 26.

28 J. M. Haschke, T. H. Allen and J. L. Stakebake, J. Alloys Compd., 1996, 243, 23-35.

29 J. M. Haschke and J. C. Martz, Los Alamos Sci., 2000, 26.

30 D. W. Osborne and E. F. W. Jr., J. Chem. Phys., 1953, 21, 1884-1887.

31 P. Hohenberg and W. Kohn, Phys. Rev., 1964, 136, B864-B871.

32 W. Kohn and L. J. Sham, Phys. Rev., 1965, 140, A1133-A1138.

33 J. T. Pegg, X. Aparicio-Anglès, M. Storr and N. H. de Leeuw, J. Nucl. Mater., 2017, 492, 269-278.

34 H. Wang and K. Konashi, J. Alloys Compd., 2012, 533, 53-57.

35 J. P. Perdew and A. Zunger, Phys. Rev. B: Condens. Matter Mater. Phys., 1981, 23, 5048-5079.

36 S. L. Dudarev, G. A. Botton, S. Y. Savrasov, C. J. Humphreys and A. P. Sutton, Phys. Rev. B: Condens. Matter Mater. Phys., 1998, 57, 1505-1509.

37 A. I. Liechtenstein, V. I. Anisimov and J. Zaanen, Phys. Rev. B: Condens. Matter Mater. Phys., 1995, 52, R5467-R5470.

38 V. I. Anisimov, J. Zaanen and O. K. Andersen, Phys. Rev. B: Condens. Matter Mater. Phys., 1991, 44, 943-954.

39 A. Georges, G. Kotliar, W. Krauth and M. J. Rozenberg, Rev. Mod. Phys., 1996, 68, 13.

40 C. Adamo and V. Barone, J. Chem. Phys., 1999, 110, 6158-6170.

41 J. Heyd, G. E. Scuseria and M. Ernzerhof, J. Chem. Phys., 2003, 118, 8207-8215.

42 I. D. Prodan, G. E. Scuseria and R. L. Martin, Phys. Rev. B: Condens. Matter Mater. Phys., 2007, 76, 033101.

43 M.-T. Suzuki, H. Ikeda and P. M. Oppeneer, J. Phys. Soc. Jpn., 2018, 87, 041008. 
44 X.-D. Wen, R. L. Martin, L. E. Roy, G. E. Scuseria, S. P. Rudin, E. R. Batista, T. M. Mccleskey, B. L. Scott, E. Bauer and J. J. Joyce, J. Chem. Phys., 2012, 137, 154707.

45 J. Kolorenč, A. B. Shick and A. I. Lichtenstein, Phys. Rev. B: Condens. Matter Mater. Phys., 2015, 92, 085125.

46 Q. Yin, A. Kutepov, K. Haule, G. Kotliar, S. Y. Savrasov and W. E. Pickett, Phys. Rev. B: Condens. Matter Mater. Phys., 2011, 84, 195111.

47 Q. Yin and S. Y. Savrasov, Phys. Rev. Lett., 2008, 100, 225504.

48 M. T. Suzuki, N. Magnani and P. M. Oppeneer, Phys. Rev. B: Condens. Matter Mater. Phys., 2013, 88, 195146.

49 D. Gryaznov, E. Heifets and D. Sedmidubsky, Phys. Chem. Chem. Phys., 2010, 12, 12273-12278.

50 P. S. Ghosh, N. Kuganathan, A. Arya and R. W. Grimes, Phys. Chem. Chem. Phys., 2018, 20, 18707-18717.

51 X.-D. Wen, R. L. Martin, G. E. Scuseria, S. P. Rudin and E. R. Batista, J. Phys. Chem. C, 2013, 117, 13122-13128.

52 I. D. Prodan, G. E. Scuseria and R. L. Martin, Phys. Rev. B: Condens. Matter Mater. Phys., 2006, 73, 045104.

53 D. A. Andersson, J. Lezama, B. P. Uberuaga, C. Deo and S. D. Conradson, Phys. Rev. B: Condens. Matter Mater. Phys., 2009, 79, 024110.

54 Y. Yang, B. Wang and P. Zhang, J. Nucl. Mater., 2013, 433, 345-350.

55 B. Sun, H. Liu, H. Song, G. Zhang, H. Zheng, X. Zhao and P. Zhang, J. Nucl. Mater., 2012, 426, 139-147.

56 P. Zhang, B.-T. Wang and X.-G. Zhao, Phys. Rev. B: Condens. Matter Mater. Phys., 2010, 82, 144110.

57 D. Gryaznov, S. Rashkeev, E. Kotomin, E. Heifets and Y. Zhukovskii, Nucl. Instrum. Methods Phys. Res., Sect. B, 2010, 268, 3090-3094.

58 G. Jomard, B. Amadon, F. Bottin and M. Torrent, Phys. Rev. B: Condens. Matter Mater. Phys., 2008, 78, 075125.

59 G. Jomard and F. Bottin, Phys. Rev. B: Condens. Matter Mater. Phys., 2011, 84, 195469.

60 X.-D. Wen, R. L. Martin, T. M. Henderson and G. E. Scuseria, Chem. Rev., 2013, 113, 1063-1096.

61 J. Boettger and A. Ray, Int. J. Quantum Chem., 2002, 90, 1470-1477.

62 S. A. Moten, R. Atta-Fynn, A. K. Ray and M. N. Huda, J. Nucl. Mater., 2016, 468, 37-45.

63 B. Dorado and P. Garcia, Phys. Rev. B: Condens. Matter Mater. Phys., 2013, 87, 195139.

64 A. Shick, J. Kolorenč, L. Havela, T. Gouder and R. Caciuffo, Phys. Rev. B: Condens. Matter Mater. Phys., 2014, 89, 041109.

65 J. B. Comly, J. Appl. Phys., 1968, 39, 716-718.

66 R. Caciuffo, G. Amoretti, P. Santini, G. H. Lander, J. Kulda and P. D. V. Du Plessis, Phys. Rev. B: Condens. Matter Mater. Phys., 1999, 59, 13892-13900.

67 W. M. Jones and E. A. L. Joseph Gordon, J. Chem. Phys., 1952, 20.

68 J. J. Huntzicker and E. F. Westrum, J. Chem. Thermodyn., 1971, 3, 61-76.

69 B. Frazer, G. Shirane, D. Cox and C. Olsen, Phys. Rev., 1965, 140, A1448.

70 B. Frazer, G. Shirane, D. Cox and C. Olsen, J. Appl. Phys., 1966, 37, 1386.
71 J. Faber, G. H. Lander and B. R. Cooper, Phys. Rev. Lett., 1975, 35, 1770-1773.

72 S. B. Wilkins, R. Caciuffo, C. Detlefs, J. Rebizant, E. Colineau, F. Wastin and G. H. Lander, Phys. Rev. B: Condens. Matter Mater. Phys., 2006, 73, 060406.

73 S. B. Wilkins, J. A. Paixão, R. Caciuffo, P. Javorsky, F. Wastin, J. Rebizant, C. Detlefs, N. Bernhoeft, P. Santini and G. H. Lander, Phys. Rev. B: Condens. Matter Mater. Phys., 2004, 70, 214402.

74 J. Faber and G. H. Lander, Phys. Rev. B: Solid State, 1976, 14, 1151-1164.

75 O. G. Brandt and C. T. Walker, Phys. Rev., 1968, 170, 528-541.

76 B. Dorado, G. Jomard, M. Freyss and M. Bertolus, Phys. Rev. B: Condens. Matter Mater. Phys., 2010, 82, 035114.

77 E. Blackburn, R. Caciuffo, N. Magnani, P. Santini, P. J. Brown, M. Enderle and G. H. Lander, Phys. Rev. B: Condens. Matter Mater. Phys., 2005, 72, 184411.

78 S. W. Yu, J. G. Tobin, J. C. Crowhurst, S. Sharma, J. K. Dewhurst, P. Olalde-Velasco, W. L. Yang and W. J. Siekhaus, Phys. Rev. B: Condens. Matter Mater. Phys., 2011, 83, 165102.

79 J. Schoenes, J. Appl. Phys., 1978, 49, 1463-1465.

80 F. Jollet, T. Petit, S. Gota, N. Thromat, M. Gautier-Soyer and A. Pasturel, J. Phys.: Condens. Matter, 1997, 9, 9393.

81 B. W. Veal and D. J. Lam, Phys. Lett. A, 1974, 49, 466-468.

82 B. W. Veal and D. J. Lam, Phys. Rev. B: Solid State, 1974, 10, 4902-4908.

83 Y. Baer and J. Schoenes, Solid State Commun., 1980, 33, 885-888.

84 Y. Baer, Physica B+C, 1980, 102, 104-110.

85 L. E. Cox, W. P. Ellis, R. D. Cowan, J. W. Allen, S. J. Oh, I. Lindau, B. B. Pate and A. J. Arko, Phys. Rev. B: Condens. Matter Mater. Phys., 1987, 35, 5761-5765.

86 P. Roussel, P. Morrall and S. J. Tull, J. Nucl. Mater., 2009, 385, 53-56.

87 J. C. Boettger and A. K. Ray, Int. J. Quantum Chem., 2000, 80, 824-830.

88 K. N. Kudin, G. E. Scuseria and R. L. Martin, Phys. Rev. Lett., 2002, 89, 266402.

89 T. Petit, G. Jomard, C. Lemaignan, B. Bigot and A. Pasturel, J. Nucl. Mater., 1999, 275, 119-123.

90 B. Ao, R. Qiu, H. Lu and P. Chen, J. Phys. Chem. C, 2016, 120, 18445-18451.

91 M. Blume, Phys. Rev., 1966, 141, 517-524.

92 M. R. Daniel, Phys. Lett., 1966, 22, 131-132.

93 J. M. Friedt, F. J. Litterst and J. Rebizant, Phys. Rev. B: Condens. Matter Mater. Phys., 1985, 32, 257-263.

94 P. Erdös, G. Solt, G. Zołnierek, A. Blaise and J. M. Fournier, Physica $B+C, 1980,102,164-170$.

95 E. F. W. Jr., J. B. Hatcher and D. W. Osborne, J. Chem. Phys, 1953, 21, 419-423.

96 D. Mannix, G. H. Lander, J. Rebizant, R. Caciuffo, N. Bernhoeft, E. Lidström and C. Vettier, Phys. Rev. B: Condens. Matter Mater. Phys., 1999, 60, 15187-15193.

97 Y. Tokunaga, Y. Homma, S. Kambe, D. Aoki, H. Sakai, E. Yamamoto, A. Nakamura, Y. Shiokawa, R. E. Walstedt and H. Yasuoka, Phys. Rev. Lett., 2005, 94, 137209. 
98 R. Caciuffo, G. H. Lander, J. C. Spirlet, J. M. Fournier and W. F. Kuhs, Solid State Commun., 1987, 64, 149-152.

99 W. Kopmann, F. J. Litterst, H. H. Klauß, M. Hillberg, W. Wagener, G. M. Kalvius, E. Schreier, F. J. Burghart, J. Rebizant and G. H. Lander, J. Alloys Compd., 1998, 271-273, 463-466.

100 P. W. Anderson, Phys. Rev., 1950, 79, 350-356.

101 W. J. De Haas, E. C. Wiersma and H. A. Kramers, Physica, 1934, 1, 1-13.

102 C. B. Finch and G. W. Clark, J. Cryst. Growth, 1970, 6, 245-248.

103 J. C. Spirlet, E. Bednarczyk, C. Rijkeboer, C. Rizzoli, J. Rebizant and O. Vogt, Inorg. Chim. Acta, 1984, 94, 111-112.

104 G. Amoretti, A. Blaise, R. Caciuffo, D. Di Cola, J. Fournier, M. Hutchings, G. Lander, R. Osborn, A. Severing and A. Taylor, J. Phys.: Condens. Matter, 1992, 4, 3459.

105 R. Caciuffo, G. Amoretti, J. M. Fournier, A. Blaise, R. Osborn, A. D. Taylor, J. Larroque and M. T. Hutchings, Solid State Commun., 1991, 79, 197-200.

106 C. Wang, M. Norman, R. Albers, A. Boring, W. Pickett, H. Krakauer and N. Christensen, Phys. Rev. B: Condens. Matter Mater. Phys., 1987, 35, 7260.

107 J. A. Mydosh and P. M. Oppeneer, Philos. Mag., 2014, 94, 3642-3662.

108 P. Santini, G. Amoretti, R. Caciuffo, F. Bourdarot and B. Fåk, Phys. Rev. Lett., 2000, 85, 654-657.

109 M. Colarieti-Tosti, O. Eriksson, L. Nordström, J. Wills and M. S. S. Brooks, Phys. Rev. B: Condens. Matter Mater. Phys., 2002, 65, 195102.

110 S. Kern, C. K. Loong, G. L. Goodman, B. Cort and G. H. Lander, J. Phys.: Condens. Matter, 1990, 2, 1933.

111 G. Raphael and R. Lallement, Solid State Commun., 1968, 6, 383-385.

112 H. Yasuoka, G. Koutroulakis, H. Chudo, S. Richmond, D. K. Veirs, A. I. Smith, E. D. Bauer, J. D. Thompson, G. D. Jarvinen and D. L. Clark, Science, 2012, 336, 901-904.

113 V. I. Anisimov, Strong coulomb correlations in electronic structure calculations, CRC Press, 2000.

114 W. H. Press, S. A. Teukolsky, W. T. Vetterling and B. P. Flannery, Numerical Recipes: The Art of Scientific Computing, Cambridge University Press, 3rd edn, 2007.
115 K. Momma and F. Izumi, J. Appl. Crystallogr., 2011, 44, 1272-1276.

116 A. V. Krukau, O. A. Vydrov, A. F. Izmaylov and G. E. Scuseria, J. Chem. Phys., 2006, 125, 224106.

117 H. J. Monkhorst and J. D. Pack, Phys. Rev. B: Solid State, 1976, 13, 5188.

118 E. Bousquet and N. Spaldin, Phys. Rev. B: Condens. Matter Mater. Phys., 2010, 82, 220402.

119 G. I. Csonka, J. P. Perdew, A. Ruzsinszky, P. H. Philipsen, S. Lebègue, J. Paier, O. A. Vydrov and J. G. Ángyán, Phys. Rev. B: Condens. Matter Mater. Phys., 2009, 79, 155107.

120 P. E. Blöchl, O. Jepsen and O. K. Andersen, Phys. Rev. B: Condens. Matter Mater. Phys., 1994, 49, 16223.

121 T. T. Meek, B. von Roedern, P. G. Clem and R. J. Hanrahan Jr, Mater. Lett., 2005, 59, 1085-1088.

122 B. Amadon, T. Applencourt and F. Bruneval, Phys. Rev. B: Condens. Matter Mater. Phys., 2014, 89, 125110.

123 T. Bo, J.-H. Lan, C.-Z. Wang, Y.-L. Zhao, C.-H. He, Y.-J. Zhang, Z.-F. Chai and W.-Q. Shi, J. Phys. Chem. C, 2014, 118, 21935-21944.

124 T. Bo, J.-H. Lan, Y.-L. Zhao, Y.-J. Zhang, C.-H. He, Z.-F. Chai and W.-Q. Shi, J. Nucl. Mater., 2014, 454, 446-454.

125 J. A. Paixão, C. Detlefs, M. J. Longfield, R. Caciuffo, P. Santini, N. Bernhoeft, J. Rebizant and G. H. Lander, Phys. Rev. Lett., 2002, 89, 187202.

126 T. M. McCleskey, E. Bauer, Q. Jia, A. K. Burrell, B. L. Scott, S. D. Conradson, A. Mueller, L. Roy, X. Wen and G. E. Scuseria, J. Appl. Phys., 2013, 113, 013515.

127 C. Suzuki, T. Nishi, M. Nakada, M. Akabori, M. Hirata and Y. Kaji, J. Phys. Chem. Solids, 2012, 73, 209-216.

128 R. Caciuffo, J. A. Paixão, C. Detlefs, M. J. Longfield, P. Santini, N. Bernhoeft, J. Rebizant and G. H. Lander, J. Phys.: Condens. Matter, 2003, 15, S2287.

129 P. Santini and G. Amoretti, Phys. Rev. Lett., 2000, 85, 2188-2191.

130 S. W. Lovesey, E. Balcar, C. Detlefs, G. V. D. Laan, D. S. Sivia and U. Staub, J. Phys.: Condens. Matter, 2003, 15, 4511.

131 J. T. Pegg, A Noncollinear Relativistic Computational Study of the Actinide Dioxides and their Interaction with Hydrogen, UCL (University College London), 2018.

132 J. T. Pegg, A. E. Shields, M. T. Storr, D. O. Scanlon and N. H. de Leeuw, J. Phys. Chem. C, DOI: 10.1021/acs.jpcc.8b07823. 